\title{
Urea Metal-Organic Frameworks as Effective and Size-Selective Hydrogen-Bond Catalysts
}

\author{
John M. Roberts, ${ }^{\dagger, \dagger}$ Branden M. Fini, ${ }^{\dagger, \dagger}$ Amy A. Sarjeant, ${ }^{\dagger}$ Omar K. Farha, ${ }^{*}, \dagger, \S$ Joseph T. Hupp, ${ }^{*},, \S$ \\ and Karl A. Scheidt $*, \dagger, \S, \ddagger$
}

${ }^{\dagger}$ Department of Chemistry, ${ }^{\S}$ International Institute for Nanotechnology, and ${ }^{\ddagger}$ Center for Molecular Innovation and Drug Discovery, Northwestern University, 2145 Sheridan Road, Evanston, Illinois 60208, United States

\section{Supporting Information}

ABSTRACT: A new urea-containing metal-organic framework (MOF) was synthesized to act as a heterogeneous catalyst. Ureas are well-known for self-recognition and aggregation behavior, resulting in loss of catalytic competency. The catalyst spatial isolation achievable in a porous MOF environment suggests a potentially general solution. The combination of a symmetrical urea tetracarboxylate strut, 4,4'-bipyridine, and $\mathrm{Zn}\left(\mathrm{NO}_{3}\right)_{2} \cdot 6 \mathrm{H}_{2} \mathrm{O}$ under solvothermal conditions afforded a new microporous MOF (NU-601). This material is indeed an effective hydrogen-bond-donor catalyst for Friedel-Crafts reactions between pyrroles and nitroalkenes, whereas a homogeneous urea is much less competent. The higher rates of reaction of small substrates relative to larger ones with NU-601 strongly suggest that catalysis primarily occurs within the pores of this new material rather than on its exterior. To the best of our knowledge, this approach is the first example of specific engineering of successful hydrogen-bonding catalysis into a MOF material.

$\mathrm{C}$ oordination polymers, including metal-organic frameworks (MOFs), have recently emerged as promising new materials ${ }^{1}$ for gas storage, ${ }^{2}$ gas separation, ${ }^{3}$ sensing, ${ }^{4,3 b}$ drug delivery, ${ }^{5}$ light harvesting, ${ }^{6}$ and heterogeneous catalysis. MOFs' tunable nature, permanent microporosity, and defined reaction environments make them ideal candidates for new directions in catalyst design and application. On the basis of our collective interests in MOFs and catalysis, we have developed a collaborative program aimed at merging metal-free (organocatalytic) and heterogeneous catalysts. ${ }^{8}$ Recently, we hypothesized that incorporating homogeneous catalysts that are prone to self-quenching into MOFs might lead to a new class of materials with significant potential. "Self-quenching" here is defined as unproductive self-assembly behavior due to catalyst-catalyst interactions (e.g., dimerization, oligomerization, etc.). This phenomenon is present in certain homogeneous systems, leading to a potential decrease in catalyst reactivity. By rendering the possibility of self-quenching unlikely, if not impossible, through spatial control of reactive catalysts centers engendered by MOF materials, new heterogeneous catalysts could potentially show increased and/or different reactivity in comparison with similar homogeneous catalysts. Such materials could also exhibit size selectivity in addition to being robust and reusable catalysts relative to their homogeneous counterparts.
Hydrogen-bond-donating (HBD) catalysis has emerged as a biomimetic alternative to Lewis acid activation. ${ }^{9}$ These reactions usually employ a urea or thiourea as the key functional group capable of two-point hydrogen bonding through acidic $\mathrm{N}-\mathrm{H}$ bonds. Numerous bond-forming reactions (e.g., Strecker reactions, ${ }^{10}$ Diels-Alder reactions, ${ }^{11}$ Claisen rearrangements, ${ }^{12}$ Mannich reactions, ${ }^{13}$ Friedel-Crafts reactions, ${ }^{14}$ conjugate additions, ${ }^{15}$ etc.) can be catalyzed by homogeneous HBD catalysts, often in excellent yield and selectivity. However, the competency of H-bond donors present in HBD catalysts such as ureas can be significantly attenuated as a result of dimerization and oligomerization through hydrogen bonding of catalyst molecules to each other. ${ }^{16} \mathrm{HBD}$ catalysts are prime examples of catalysts that undergo self-quenching, and these unproductive interactions greatly decrease the catalyst solubility and reactivity (Figure 1 top). Consequently, new directions and opportunities

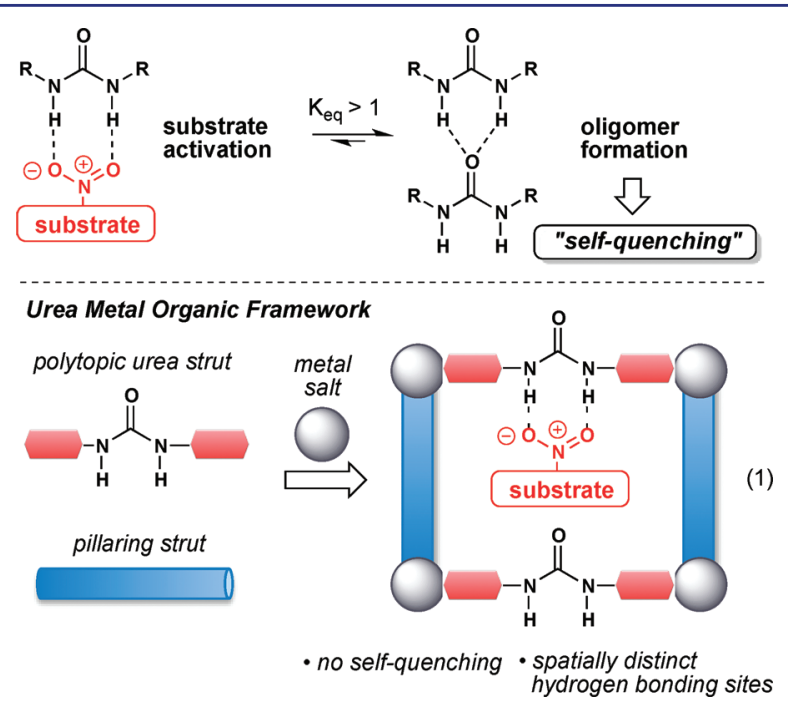

Figure 1. Urea MOF strategy.

might be realized if these catalysts were to be incorporated into coordination polymers, which possess defined reaction environments and high porosity. The combination of a polytopic urea "strut", a pillaring strut, and an appropriate metal ion could produce materials that avoid the possibility of self-quenching

Received: November 16, 2011

Published: January 31, 2012 
while possessing spatially distinct hydrogen-bonding sites (eq 1 in Figure 1). Herein we report the synthesis and initial application of a new urea-containing MOF designed for heterogeneous HBD catalysis following the principles described above. ${ }^{17}$

We based our design of $\mathrm{H}$-bond MOF strut 1 on a symmetrical thiourea developed by Schreiner and co-workers (Figure 2). ${ }^{11}$ Thermal stability issues that we encountered with

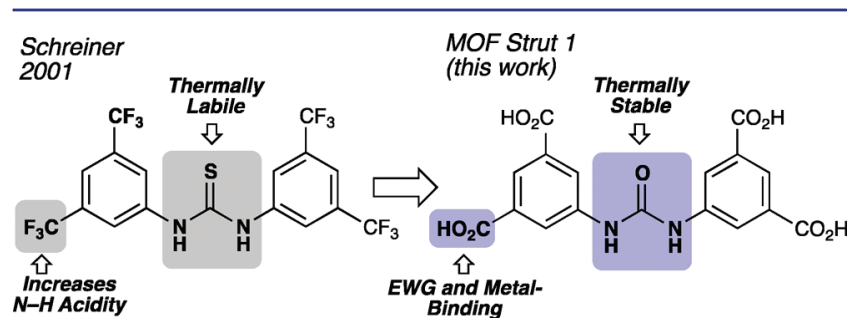

Figure 2. Urea MOF strut design.

thioureas under standard MOF solvothermal conditions led us to instead use the corresponding ureas, given their strong propensity to act as hydrogen-bond donors. ${ }^{12}$ The $\mathrm{CF}_{3}$ groups present in these urea/thiourea catalysts were replaced with carboxylic acids, which serve a secondary and critical design criterion, as they are metal-binding groups. Strut $\mathbf{1}$ was produced efficiently from 5-nitroisophthalic acid in four steps with an overall yield of $50 \%$ [see the Supporting Information (SI)], allowing easy access to multigram quantities of this material. With the strut in hand, we set out to make a material with pores large enough to accommodate substrates for HBD-catalyzed reactions.

We initially sought to synthesize MOFs containing 2D sheets of ureas connected by zinc paddlewheel secondary building units and pillared with 4,4'-bipyridine (BIPY) (Scheme 1).

Scheme 1. (top) Synthesis of NU-601; (bottom) Views of (A) 1 and the $\mathrm{Zn}_{2}$ Nodes and (B) the Repeating Unit of NU-601 ${ }^{a}$
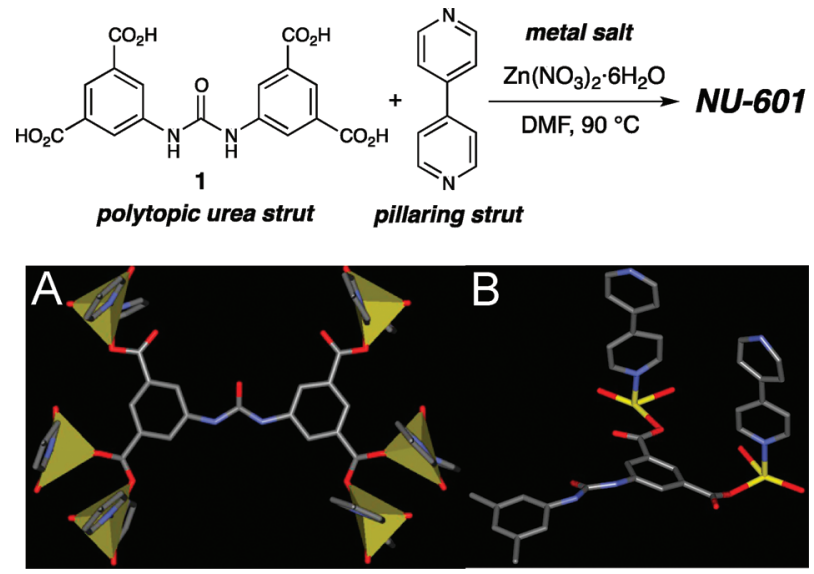

${ }^{a}$ In the bottom panel, $\mathrm{H}$ atoms have been omitted for clarity. The view in (A) shows that in each of the two benzenedicarboxylate groups of $\mathbf{1}$, one of the carboxylates coordinates to one $\mathrm{Zn}$ atom and the other coordinates to two $\mathrm{Zn}$ atoms.

After 2 days of heating under solvothermal conditions, we observed light-yellow needle-shaped crystals having the formula $\mathrm{Zn}_{2}(\mathrm{BIPY})_{2}(1-4 \mathrm{H})$, which we have designated NU-601. These crystals were subjected to single-crystal X-ray diffraction (XRD) to elucidate their structure. XRD revealed that NU-601 does in fact contain $2 \mathrm{D}$ sheets of strut $\mathbf{1}$ pillared with BIPY. This material contains $\mathrm{Zn}_{2}$ nodes similar to those in a material reported by $\mathrm{Li}$ and co-workers. ${ }^{18}$

NU-601 exists in the $P 2_{1} / c$ space group. It possesses large channels in all directions ( $a$ axis, $12.05 \AA \times 13.95 \AA$; $b$ axis, $11.38 \AA \times 13.69 \AA$; $c$ axis, $11.38 \AA \times 4.87 \AA$; distances measured between atom centers) with $\mathrm{N}-\mathrm{H}$ urea bonds projecting into the pores (Figure 3). As anticipated, the oxygen atoms of

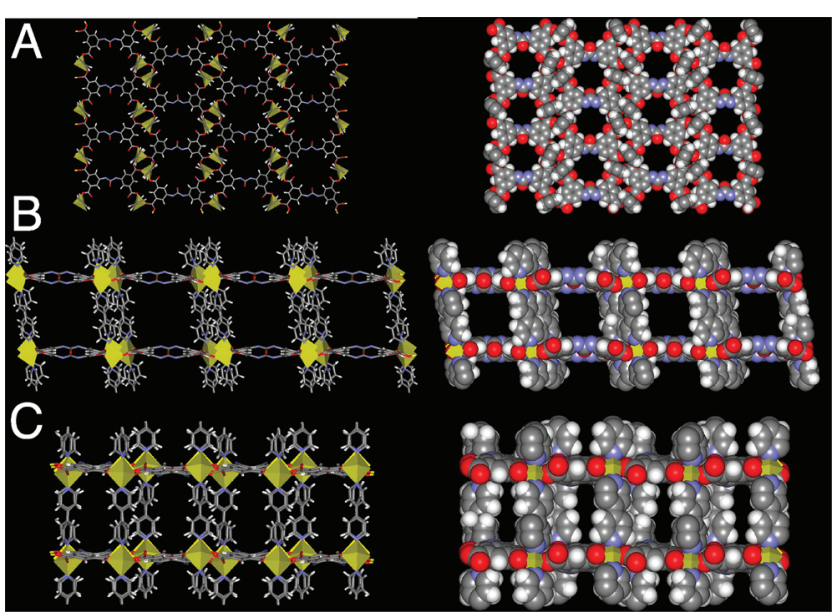

Figure 3. Stick and space-filling depictions of NU-601: (A) view down the $a$ axis; (B) view down the $b$ axis; (C) view down the $c$ axis.

the remaining $N, N$-dimethylformamide (DMF) molecules are tightly hydrogen-bonded to the urea $\mathrm{N}-\mathrm{H}$ bonds in the solidstate structure. Powder XRD (PXRD) data from bulk samples of NU-601 matched the simulated PXRD pattern (generated from the CIF file; see the SI). To open the $\mathrm{N}-\mathrm{H}$ urea sites and render NU-601 catalytically active, it was necessary to remove the residual hydrogen-bonded DMF. Thermogravimetric analysis (TGA) of NU-601 revealed $\sim 30 \%$ weight loss after heating to $125{ }^{\circ} \mathrm{C}$ (see the SI). Another weight loss event was observed beginning at $\sim 200{ }^{\circ} \mathrm{C}$, which we attribute to the loss of hydrogen-bonded DMF. However, the PXRD pattern of this heated material indicated a loss of crystallinity, and additional standard thermal strategies proved unsuccessful in removing all of the DMF. The crucial activation was accomplished by exchanging the DMF with $\mathrm{MeNO}_{2}$ over a period of 4 days. The MOF was then digested in $\mathrm{D}_{2} \mathrm{SO}_{4}$, and ${ }^{1} \mathrm{H}$ NMR spectroscopic analysis confirmed that DMF had been $>95 \%$ replaced with $\mathrm{MeNO}_{2} .{ }^{1} \mathrm{H}$ NMR spectroscopy $(500 \mathrm{MHz})$ also allowed us to determine the percentage by weight of strut 1 in NU-601 $[\sim 36 \%(\mathrm{w} / \mathrm{w}) \mathbf{1}$; see the SI], which was necessary to calculate the catalyst loading. Solvent exchange (vs thermal activation) has proved to be a general and effective method of preparing MOFs for solution-phase catalysis reactions, especially when thermal activation is problematic.

The next goal was to determine whether the urea-containing NU-601 after $\mathrm{MeNO}_{2}$ exchange/activation could function as an HBD catalyst. Given NU-601's medium pore size, it was necessary to model substrates and products from known HBD reactions $^{12,10,11,13}$ using MM2 to predict whether these molecules could penetrate the MOF structure. The Friedel-Crafts reaction between pyrroles and nitroalkenes ${ }^{19}$ was identified as a potential test for NU-601, since the substrates appeared to be accommodated by the pores and the corresponding homogeneous reaction employs a urea catalyst similar to strut $\mathbf{1}^{14}$ During this in silico evaluation, we also determined that any 
Friedel-Crafts products between pyrroles and nitroalkenes bound to a urea site within NU-601 (directly after carboncarbon bond formation) would be large enough to preclude access to a second adjacent site, thereby blocking catalysis by $\sim 50 \%$ of the ureas within the MOF (see the SI). The initial experiments to catalyze the Friedel-Crafts reaction between $\mathrm{N}$-methylpyrrole (5) and (E)-1-nitroprop-1-ene (6) with $10 \mathrm{~mol} \%$ NU-601 (0.1 $\mathrm{M}$ in toluene at $23{ }^{\circ} \mathrm{C}$; Figure 4$)$ afforded no

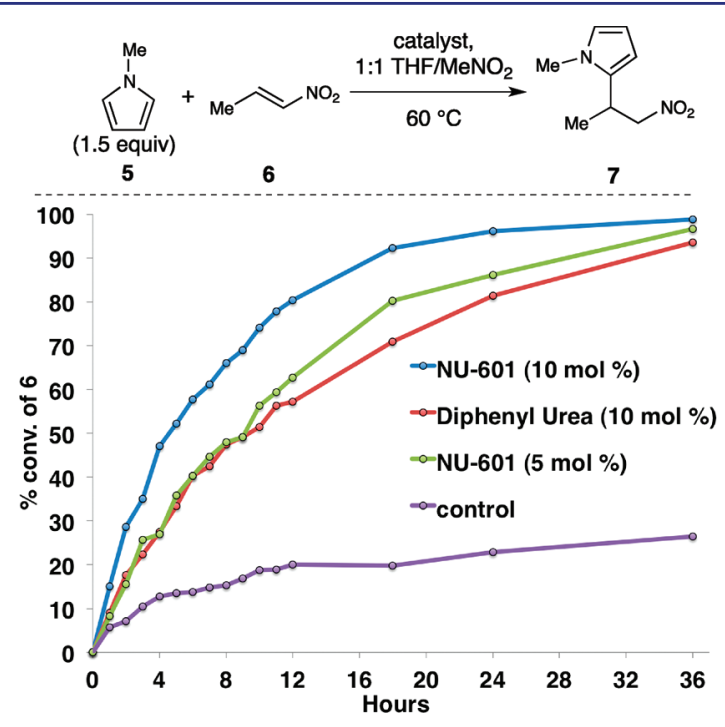

Figure 4. Catalytic activities of NU-601, diphenylurea, and a control. All of the reactions were performed for $36 \mathrm{~h}$ and monitored by GC using mesitylene as an internal standard.

consumption of 6 as observed by GC (data not shown). This result was attributed to product inhibition in the nonpolar solvent, which is used in homogeneous systems to increase the catalyst-substrate interactions (i.e., the overall reaction rate). To promote catalyst turnover and product displacement by substrates in the more challenging heterogeneous system, the reaction solvent (toluene) was changed to a more polar mixture (1:1 $\left.\mathrm{MeNO}_{2} / \mathrm{THF}\right)$ to potentially facilitate more $\mathrm{H}$-bonding exchange. In addition, the reaction concentration and temperature were increased to $1.0 \mathrm{M}$ and $60{ }^{\circ} \mathrm{C}$. Under these new reaction conditions, $98 \%$ consumption of 6 was observed after $36 \mathrm{~h}$.

Next, we compared NU-601 to diphenylurea, a homogeneous urea (under our reaction conditions) prone to oligomerization/ self-quenching as observed in the solid state. ${ }^{16}$ As shown in Figure 4, NU-601 is a more active catalyst than diphenylurea for the Friedel-Crafts reaction (90\% conv. at $18 \mathrm{~h}$ vs $65 \%$ conv.). These overall rate data validate the hypothesis that encapsulating a urea catalyst within a MOF leads to improved reactivity. Indeed, even when NU-601 was used at $5 \mathrm{~mol} \%$ catalyst loading (which assumes every catalyst site is available), it compared favorably to diphenylurea ( $80 \%$ conv. at $18 \mathrm{~h}$ vs $68 \%$ conv.). Importantly, NU-601 showed a significantly higher rate of substrate consumption versus the control reaction. We then sought to confirm that the active catalyst species did not leach into solution by performing the reaction with removal of NU-601 after $6 \mathrm{~h}$. This elicited a sharp decrease in the rate, which is strong evidence that the reaction is heterogeneous in nature (see the SI). NU-601 also proved to be reusable, maintaining its reactivity after one cycle of use and experiencing only a slight degradation of reactivity after five cycles while retaining its crystallinity as determined by PXRD (see the SI).

We next investigated whether the catalysis occurred on the surface or within the pores of NU-601 by using larger substrates as steric probes (Scheme 2). Toward this end, when

Scheme 2. Size-Exclusion Catalysis Experiments (Reactions Performed with $5 \mathrm{~mol} \%$ NU-601 for $48 \mathrm{~h}$ )

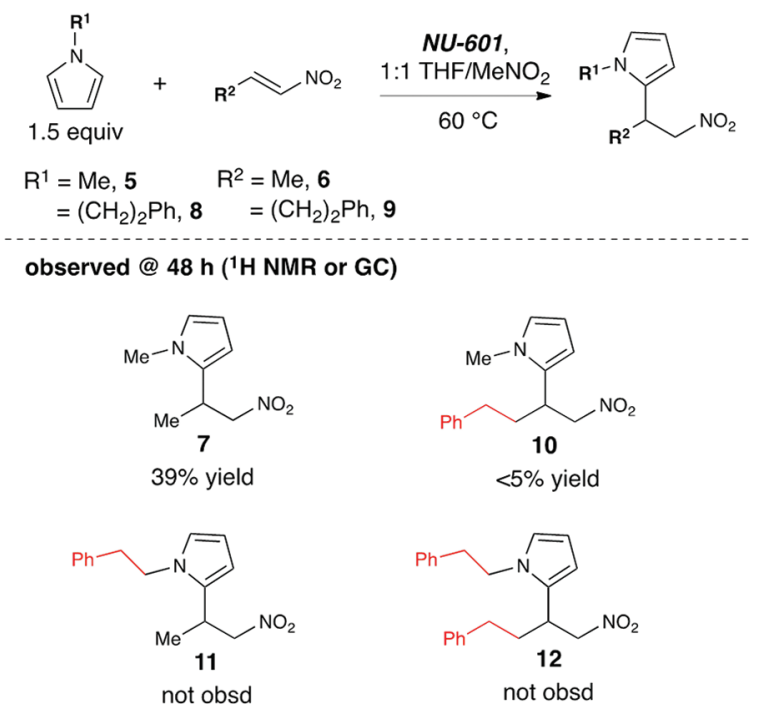

1-phenethyl-1H-pyrrole (8) and/or (E)-(4-nitrobut-3-en-1-yl)benzene (9) were employed, the substrate consumption and reaction yield decreased rapidly. These observations provide strong evidence that NU-601 exhibits reagent size selectivity, since large and small reagents show similar reactivities in related homogeneous systems (see the SI). Another key result is that while an observable amount $(<5 \%)$ of Friedel-Crafts product 10 was detected when nitroalkene 9 was used with 5, no Friedel-Crafts products were observed when pyrrole 8 was used, suggesting that large substrates, especially pyrroles, cannot enter the porous network. To investigate further the size selectivity of NU-601, we performed a competition reaction by combining 6 and the larger conjugate acceptor 9 ( 1 equiv each) with 5 under the standard urea MOF conditions using $5 \mathrm{~mol} \%$ NU-601. While both products 7 and 10 were possible, especially in a homogeneous approach, we were pleased to only observe 7 , albeit in a diminished yield (see the SI).

In summary, a novel urea-containing MOF was synthesized from a tetracarboxylate strut (1), 4,4'-bipyridine, and $\mathrm{Zn}\left(\mathrm{NO}_{3}\right)_{2}$. $6 \mathrm{H}_{2} \mathrm{O}$. This new material is a competent and size-selective hydrogen-bond-donor catalyst for Friedel-Crafts reactions. When NU-601 is used, large substrates show significantly diminished yields versus small substrates, strongly supporting the conclusion that catalysis occurs mainly within the pores. This new heterogeneous organocatalyst also shows an increased rate enhancement versus diphenylurea, which presumably is limited in reactivity as a catalyst because of self-quenching behavior. To the best of our knowledge, this approach is the first example of hydrogen-bonding catalysis in a MOF. Efforts are currently underway in our laboratories to synthesize MOFbased HBD catalysts that are more highly active and to design other MOF-based heterogeneous systems that provide different characteristics than corresponding homogeneous systems. 


\section{ASSOCIATED CONTENT}

\section{S Supporting Information}

Detailed experimental procedures; NMR, MS, IR, TGA, PXRD, and reaction data; and crystallographic data (CIF). This material is available free of charge via the Internet at http://pubs.acs.org.

\section{AUTHOR INFORMATION}

\section{Corresponding Author}

o-farha@northwestern.edu; j-hupp@northwestern.edu; scheidt@nothwestern.edu

Notes

The authors declare no competing financial interest.

\section{ACKNOWLEDGMENTS}

K.A.S. thanks Amgen for generous support and AFOSR for initial support of this work. J.M.R and B.M.F. thank the Bordwell Chemistry Research Fund (NU) and a Northwestern Summer URG program, respectively, for partial fellowship support. O.K.F. and J.T.H. thank the AFOSR and DTRA (Grant HDTRA1-09-1-0007) for financial support. The authors acknowledge Chris Wilmer (NU) for assistance with modeling.

\section{REFERENCES}

(1) (a) Férey, G. Chem. Soc. Rev. 2008, 37, 191. (b) Horike, S.; Shimomura, S.; Kitagawa, S. Nat. Chem. 2009, 1, 695. (c) O'Keeffe, M.; Yaghi, O. M. Chem. Rev. 2011, DOI: 10.1021/cr200205j.

(2) (a) Murray, L. J.; Dincă, M.; Long, J. R. Chem. Soc. Rev. 2009, 38 1294. (b) Farha, O. K.; Yazaydin, A. O.; Eryazici, I.; Malliakas, C. D.; Hauser, B. G.; Kanatzidis, M. G.; Nguyen, S. T.; Snurr, R. Q.; Hupp, J. T. Nat. Chem. 2010, 2, 944. (c) Furukawa, H.; Ko, N.; Go, Y. B.; Aratani, N.; Choi, S. B.; Choi, E.; Yazaydin, A. O.; Snurr, R. Q.; O’Keeffe, M.; Kim, J.; Yaghi, O. M. Science 2010, 329, 424.

(3) (a) Thallapally, P. K.; Tian, J.; Kishan, M. R.; Fernandez, C. A.; Dalgarno, S. J.; McGrail, P. B.; Warren, J. E.; Atwood, J. L. J. Am. Chem. Soc. 2008, 130, 16842. (b) Sun, L. X.; Song, L. F.; Jiang, C. H.; Jiao, C. L.; Zhang, J. A.; Xu, F.; You, W. S.; Wang, Z. G.; Zhao, J. J. Cryst. Growth Des. 2010, 10, 5020.

(4) Achmann, S.; Hagen, G.; Kita, J.; Malkowsky, I. M.; Kiener, C.; Moos, R. Sensors 2009, 9, 1574.

(5) Morris, R. E.; McKinlay, A. C.; Horcajada, P.; Férey, G.; Gref, R.; Couvreur, P.; Serre, C. Angew. Chem., Int. Ed. 2010, 49, 6260.

(6) (a) Kent, C. A.; Liu, D.; Ma, L.; Papanikolas, J. M.; Meyer, T. J.; Lin, W. J. Am. Chem. Soc. 2011, 133, 12940. (b) Lee, C. Y.; Farha, O. K.; Hong, B. J.; Sarjeant, A. A.; Nguyen, S. T.; Hupp, J. T. J. Am. Chem. Soc. 2011, 133, 15858 .

(7) Lee, J.; Farha, O. K.; Roberts, J.; Scheidt, K. A.; Nguyen, S. T.; Hupp, J. T. Chem. Soc. Rev. 2009, 38, 1450.

(8) (a) Lee, J. Y.; Roberts, J. M.; Farha, O. K.; Sarjeant, A. A.; Scheidt, K. A.; Hupp, J. T. Inorg. Chem. 2009, 48, 9971. (b) Roberts, J. M.; Farha, O. K.; Sarjeant, A. A.; Hupp, J. T.; Scheidt, K. A. Cryst. Growth Des. 2011, 11, 4747. For recent examples of metal-centric MOF-based catalysis from Northwestern University, see: (c) Shultz, A. M.; Sarjeant, A. A.; Farha, O. K.; Hupp, J. T.; Nguyen, S. T. J. Am. Chem. Soc. 2011, 133, 13252. (d) Farha, O. K.; Shultz, A. M.; Sarjeant, A. A.; Nguyen, S. T.; Hupp, J. T. J. Am. Chem. Soc. 2011, 133, 5652. (e) Shultz, A. M.; Farha, O. K.; Adhikari, D.; Sarjeant, A. A.; Hupp, J. T.; Nguyen, S. T. Inorg. Chem. 2011, 50, 3174. For recent examples of organocatalysis using MOFs, see: (f) Banerjee, M.; Das, S.; Yoon, M.; Choi, H. J.; Hyun, M. H.; Park, S. M.; Seo, G.; Kim, K. J. Am. Chem. Soc. 2009, 131, 7524. (g) Dang, D.; Wu, P.; He, C.; Xie, Z.; Duan, C. J. Am. Chem. Soc. 2010, 132, 14321. (h) Lun, D. J.; Waterhouse, G. I. N.; Telfer, S. G. J. Am. Chem. Soc. 2011, 133, 5806.

(9) For reviews, see: (a) Schreiner, P. R. Chem. Soc. Rev. 2003, 32, 289. (b) Taylor, M. S.; Jacobsen, E. N. Angew. Chem., Int. Ed. 2006, 45, 1520. (c) Jacobsen, E. N.; Doyle, A. G. Chem. Rev. 2007, 107, 5713. (d) Connon, S. J. Chem. Commun. 2008, 2499. (e) Zhang, Z.; Schreiner, P. R. Chem. Soc. Rev. 2009, 38, 1187.

(10) Sigman, M. S.; Jacobsen, E. N. J. Am. Chem. Soc. 1998, 120, 4901.

(11) Schreiner, P. R.; Wittkopp, A. Org. Lett. 2002, 4, 217.

(12) For early studies, see: (a) Etter, M. C.; Reutzel, S. M. J. Am. Chem. Soc. 1991, 113, 2586. (b) Smith, P. J.; Kim, E.-i.; Wilcox, C. S. Angew. Chem., Int. Ed. Engl. 1993, 32, 1648. (c) Fan, E.; Van Arman, S. A.; Kincaid, S.; Hamilton, A. D. J. Am. Chem. Soc. 1993, 115, 369. (d) Curran, D. P.; Kuo, L. H. J. Org. Chem. 1994, 59, 3259.

(13) Jacobsen, E. N.; Wenzel, A. G. J. Am. Chem. Soc. 2002, 124, 12964.

(14) Dessole, G.; Gabriella, D.; Herrera, R. P.; Riccia, A. Synlett 2004, 2374.

(15) Biddle, M. M.; Lin, M.; Scheidt, K. A. J. Am. Chem. Soc. 2007, 129,3830

(16) For X-ray studies of self-association of diphenylureas and related compounds, see: (a) Deshapan, S. V.; Meredith, C. C.; Pasterna, R. A. Acta Crystallogr. 1968, B24, 1396. (b) Stankovic, S.; Andreetti, G. D. Acta Crystallogr. 1978, B34, 3787. (c) George, S.; Nangia, A.; Lynch, V. M. Acta Crystallogr. 2001, C57, 777. (d) George, S.; Nangia, A. Acta Crystallogr. 2003, E59, 901. (e) Slawin, A. M. Z.; Lawson, J.; Storey, J. M. D.; Harrison, W. T. A. Acta Crystallogr. 2007, E63, 2925. (f) 3, 5-Dicarboxy-substituted arylureas (and related esters) are poor materials for comparison since these esters prevent dimerization through steric "shielding". Modeling with the aryl rings aligned in-plane with the urea carbonyl group clearly showed that the ester substituents of one urea engender destablizing/unfavorable van der Waals interactions with a second 3,5-dicarboxyurea ester before the carbonyl group of one molecule is close enough to the $\mathrm{NH}$ groups of a second urea molecule for intermolecular self-quenching.

(17) MOFs containing hydrogen-bond donors (primarily ureas) are known, but to the best of our knowledge, none have been investigated as HBD catalysts. For selected examples, see: (a) Custelcean, R; Moyer, B. A.; Hay, B. P. Chem. Commun. 2005, 5971. (b) Custelcean, R; Haverlock, T. J.; Moyer, B. A. Inorg. Chem. 2006, 45, 6446. (c) Custelcean, R.; Moyer, B. A.; Bryantsev, V. S.; Hay, B. P. Cryst. Growth Des. 2006, 6, 555. (d) Das, A.; Kumar, D. K.; Dastidar, P. New J. Chem. 2006, 30, 1267. (e) Vilar, R.; Diaz, P.; Benet-Buchholz, J.; White, A. J. P. Inorg. Chem. 2006, 45, 1617. (f) Custelcean, R.; Moyer, B. A. Eur. J. Inorg. Chem. 2007, 1321. (g) Custelcean, R.; Sellin, V.; Moyer, B. A. Chem. Commun. 2007, 1541. (h) Custelcean, R.; Remy, P.; Bonnesen, P. V.; Jiang, D. E.; Moyer, B. A. Angew. Chem., Int. Ed. 2008, 47, 1866. (i) Dastidar, P.; Adarsh, N. N.; Kumar, D. K. CrystEngComm 2008, 10, 1565. (j) Cohen, S. M.; Garibay, S. J.; Wang, Z. Q.; Tanabe, K. K. Inorg. Chem. 2009, 48, 7341. (k) Custelcean, R.; Bock, A.; Moyer, B. A. J. Am. Chem. Soc. 2010, 132, 7177. (1) Custelcean, R.; Rajbanshi, A.; Moyer, B. A. Cryst. Growth Des. 2011, 11, 2702

(18) Li, J.; Lan, A. J.; Li, K. H.; Wu, H. H.; Olson, D. H.; Emge, T. J.; Ki, W.; Hong, M. C. Angew. Chem., Int. Ed. 2009, 48, 2334.

(19) García, J. M.; Oiarbide, M.; Palomo, C.; Bartoli, G.; Melchiorre, P.; Bernardi, L.; Ricci, A. In Catalytic Asymmetric Friedel-Crafts Alkylations; Wiley-VCH: Weinheim, Germany, 2009; pp 17-99. For selected recent examples of homogeneous catalysis using pyrroles in Friedel-Crafts reactions, see: (a) Paras, N. A.; MacMillan, D. W. C. J. Am. Chem. Soc. 2001, 123, 4370. (b) Zhuang, W.; Gathergood, N.; Hazell, R. G.; Jørgensen, K. A. J. Org. Chem. 2001, 66, 1009. (c) Bonini, B. F.; Capito, E.; Comes-Franchini, M.; Fochi, M.; Riccia, A.; Zwanenburg, B. Tetrahedron: Asymmetry 2006, 17, 3135. (d) Blay, G.; Fernandez, I.; Pedro, J. R.; Vila, C. Org. Lett. 2007, 9, 2601. (e) Li, G. L.; Rowland, G. B.; Rowland, E. B.; Antilla, J. C. Org. Lett. 2007, 9, 4065. (f) Trost, B. M.; Muller, C. J. Am. Chem. Soc. 2008, 130, 2438. (g) Yokoyama, N.; Arai, T. Chem. Commun. 2009, 3285. 